\title{
Experiencia clínica con 53 trasplantes cardiacos consecutivos
}

\author{
MAURICIO VILLAVICENCIO ${ }^{1,2,3}$, VÍCTOR ROSSEL ${ }^{1,3}$, \\ RICARDO LARREA ${ }^{2}$, JUAN PABLO PERALTA ${ }^{2}$, ERNESTO LARRAÍN ${ }^{2}$, \\ JONG SUNG LIM ${ }^{1}$, PAMELA ROJO ${ }^{2}$, FRANCESCA GAJARDO ${ }^{1, a}$, \\ ERIKA DONOSO ${ }^{1, a}$, MARGARITA HURTADO ${ }^{2, a}$
}

\section{Clinical experience with 53 consecutive heart transplants}

Introduction: Heart transplantation is the therapy of choice for advance heart failure. Our group developed two transplant programs at Instituto Nacional del Tórax and Clínica Dávila. We report our clinical experience based on distinctive clinical policies. Patients and Methods: Fifty-three consecutive patients were transplanted between November 2008 and April 2013, representing 51\% of all Chilean cases. Distinctive clinical policies include intensive donor management, generic immunosuppression and VAD (ventricular assist devices) insertion. Results: Ischemic or dilated cardiomyopathy were the main indications (23 (43\%) each), age $48 \pm 13$ years and 48 (91\%) were male. Transplant listing Status: IA 14 (26\%) (VAD or 2 inotropes), IB 14 (26\%) (1 inotrope) and II 25 (47\%) (no inotrope). Mean waiting time $70 \pm$ 83 days. Twelve (24\%) were transplanted during VAD support (median support: 36 days). Operative technique: orthotopic bicaval transplant with ischemia time: $175 \pm 54$ min. Operative mortality: 3 (6\%), all due to right ventricular failure. Re-exploration for bleeding 2 (4\%), stroke $3(6 \%)$, mediastinitis $0(0 \%)$, pneumonia $4(8 \%)$, and transient dialysis $6(11 \%)$. Mean follow-up was $21 \pm 14$ months. Three-year survival was $86 \pm 6 \%$. One patient died of Pneumocystis jirovecii pneumonia and the other died suddenly (non-compliance). Freedom from rejection requiring specific therapy was $80 \pm 7 \%$ at 3 years of follow-up. Four hundred eighty four endomyocardial biopsies were done: 11 (2.3\%) had 2R rejection. All survivors are in NYHA (New York Heart Association) functional class I and all but one have normal biventricular function. Conclusion: Mid-term results are similar to those reported by the registry of the International Society for Heart and Lung Transplantation. This experience has a higher proportion of VAD support than previous national series. Rejection rates are low in spite of generic immunosuppression.

(Rev Med Chile 2013; 141: 1499-1505)

Key words: Cardiomyopathies; Heart-assist devices; Heart transplantation.

Unidad de Trasplante
Cardiopulmonar
'Instituto Nacional del
Tórax. Santiago de Chile.
${ }^{2}$ Clínica Dávila. Santiago
de Chile.
'Departamento de Cirugía
y Medicina Oriente,
Facultad de Medicina
Universidad de Chile.
'Enfermera Coordinadora
de Trasplante.
No hubo apoyo financiero.
Recibido el 24 de octubre
de 2012, aceptado el 28 de
octubre de 2013.
Correspondencia a:
Dr. Mauricio Villavicencio
Theoduloz.
Unidad de Trasplante
Cardiopulmonar, Instituto
Nacional del Tórax.
Dirección: José Miguel
Infante 717, Piso 5,
Providencia, Santiago,
Chile.
Teléfono: 562-5755020.
Fax: 562-5754853
E-mail: mvillavicencio@
torax.cl

Unidad de Trasplante

Cardiopulmonar "Instituto Nacional del ${ }^{2}$ Clínica Dávila. Santiago de Chile.

${ }^{3}$ Departamento de Cirugía y Medicina Oriente, Facultad de Medicina Universidad de Chile. aEnfermera Coordinadora de Trasplante.

No hubo apoyo financiero.

Recibido el 24 de octubre de 2012, aceptado el 28 de Correspondencia a: Dr. Mauricio Villavicencio Theoduloz. Unidad de Trasplante Cardiopulmonar, Instituto Dirección: José Migue Infante 717, Piso 5, Providencia, Santiago, Chile. Teléfono: $562-5755020$ E-mail:mvillavicencio@ torax.cl
E 1 trasplante cardiaco es la terapia de elección para la insuficiencia cardiaca avanzada. Tal como ocurre en gran parte del mundo, el número de casos de trasplante cardiaco en Chile se ha mantenido estable en los últimos años, por limitaciones en la disponibilidad de donantes. Sin embargo, en los últimos 2 años se ha producido un aumento cercano a $50 \%$ en el número de tras- plantes a nivel nacional, que puede tener que ver con cambios en la política gubernamental como también en la conducta clínica de los nuevos centros de trasplante ${ }^{1}$.

A nivel internacional, la experiencia comunicada en trasplante cardiaco alcanza los 104.000 pacientes totales y cerca de 3.700 pacientes por año en el registro de la International Society for 
Heart and Lung Transplantation (ISHLT), con una sobrevida promedio de 10 años ${ }^{2}$. La mayoría de los casos reportados son de Europa y Norteamérica. En cuanto a la experiencia publicada en Chile encontramos sólo 2 series clínicas en revistas indexadas con cerca de 25 casos cada una ${ }^{3,4}$.

En los últimos años, nuestro grupo cardioquirúrgico desarrolló 2 nuevos programas de trasplante cardiaco en el país, uno en el Instituto Nacional del Tórax y otro en Clínica Dávila. El primero se constituyó como centro de referencia para trasplante cardiaco del sistema público de salud en la Región Metropolitana. En estos dos centros se adoptó una política clínica distintiva que consistió en el manejo intensivo de donantes y la terapia con dispositivos de asistencia ventricular mecánica con el propósito de lograr un puente al trasplante. En cuanto a la inmunosupresión, se decidió incorporar la utilización de medicamentos genéricos con el fin de minimizar los costos y aumentar la cantidad de pacientes que pudieran acceder a esta terapia.

Creemos entonces importante realizar un análisis retrospectivo de los resultados que se obtuvieron en los primeros 53 trasplantes cardiacos consecutivos realizados con esta práctica clínica. También se analizó la sobrevida libre de rechazo como elemento evaluador de la inmunosupresión genérica en base a nuestro protocolo de biopsias endomiocárdicas.

\section{Pacientes y Métodos}

Análisis retrospectivo de los registros clínicos de los pacientes trasplantados del corazón consecutivamente en el período comprendido entre noviembre de 2008 y abril de 2013. El programa de la Clínica Dávila comenzó en noviembre de 2008 y el del Instituto Nacional del Tórax en diciembre de 2009. El seguimiento se completó en mayo de 2013. La indicación de trasplante cardiaco fue insuficiencia cardiaca capacidad funcional III a IV de la New York Heart Association (NYHA) con un consumo de oxígeno menor de $14 \mathrm{ml} / \mathrm{kg} / \mathrm{min}$, dependencia de drogas vasoactivas o asistencia ventricular mecánica en los pacientes que estaban en shock cardiogénico.

Los pacientes se enlistaron según los criterios establecidos por la Coordinadora Nacional de Trasplantes del Ministerio de Salud, esto es:
Estatus IA: dos o más drogas vasoactivas, ventilación mecánica y/o asistencia ventricular. Estatus IB: una droga vasoactiva. Estatus II: ausencia de requerimiento de drogas vasoactivas o asistencia ventricular. Los aparatos de asistencia ventricular mencionados (Centrimag, Heartware, Heartmate II) han sido descritos previamente $e^{5,6,7,8,9,10,11}$.

Los donantes se trataron por parte del equipo de procuramiento con corrección metabólica intensiva (hematocrito $>30 \%$, $\mathrm{pH}$ de 7,36 a 7,44, potasio de 4,0 a $5,0 \mathrm{mEq} / \mathrm{lt}$, glicemias menores a 150), drogas vasoactivas y reposición de volumen para alcanzar objetivos hemodinámicos prestablecidos (presión arterial media de 70 a 85 mmHg, PVC de 6 a 12, PCP de 12 a 15 en caso de tener Swan-Ganz) y reanimación hormonal en todos los pacientes (metilprednisolona, tiroxina endovenosa, vasopresina e insulina en bomba de infusión continua). La reanimación hormonal no es parte del tratamiento estándar efectuado por la Coordinadora Nacional de Trasplantes del Ministerio de Salud.

La técnica de procuramiento consistió en heparina sistémica $3 \mathrm{mg} / \mathrm{kg}$ endovenosa, cardiectomía con preservación del corazón con cardioplegia anterógrada cristaloide Saint Thomas II $20 \mathrm{ml} /$ $\mathrm{kg}$, hipotermia tópica con suero salino a $4^{\circ} \mathrm{C}$ y almacenamiento en 3 bolsas con suero salino frío en una hielera de transporte.

La técnica quirúrgica empleada fue un trasplante cardiaco ortotópicobicavo. Se realizó preservación miocárdica adicional una vez que el corazón donado llegó al hospital del receptor antes de liberar el clamp aórtico con cardioplegia sanguínea anterógrada a $8^{\circ} \mathrm{C}$ cada 20 min (durante las anastomosis cardiacas). En el caso de los pacientes conectados a asistencia ventricular, se prefirió entrar en circulación extracorpórea primero y luego realizar la disección del corazón dada la habitual pérdida de planos quirúrgicos.

En cuanto a la inmunosupresión, se utilizó inducción con baxiliximab (Simulect ${ }^{\circledR}$, Novartis, Basilea, Suiza) en todos los pacientes y triterapia genérica con inhibidores de la calcineurina, antimetabolitos y esteroides. En cuanto a los inhibidores de la calcineurina, en el primer tercio de la experiencia predominó el uso de ciclosporina (Transporina ${ }^{\circledR}$, Recalcine, Santiago, Chile) y en los dos tercios finales el uso de tacrolimus (T-inmun ${ }^{\circledR}$, Recalcine, Santiago, Chile). El antimetabolito de elección fue el micofenolatomofetil (Linfonex ${ }^{\circledR}$, 
Recalcine, Santiago, Chile). La azatioprina (Imu$\operatorname{ran}^{\circledR}$, Biotoscana, Santiago, Chile) se utilizó solamente cuando había contraindicación o efectos adversos con el micofenolato (ej. diarrea). El retiro de los esteroides se inició en el mes 6 en los pacientes que no habían tenido rechazo significativo. El protocolo de biopsias endomiocárdicas utilizado se basa en el del Hospital Clinic y Provincial de Barcelona: inicialmente cada 2 semanas por 3 meses, luego mensual por 3 meses más y finalmente trimestral hasta el año. Adicionalmente, se hicieron 2 biopsias post retiro de esteroides: una al mes y otra a los 3 meses. En esta serie no hubo pacientes sensibilizados contra antígenos de histocompatibilidad humana por lo que no se usó inmunoglobulina endovenosa, plasmaféresis o rituximab pre-trasplante.

Las variables categóricas fueron descritas con valor absoluto/porcentajes y las continuas con promedio/desviación estándar. La sobrevida fue estimada con el método de Kaplan-Meier más/ menos el error estándar de la media.

\section{Resultados}

Se trasplantaron 53 pacientes en forma consecutiva, 33 (62\%) en el Instituto Nacional del Tórax y 20 (38\%) en Clínica Dávila; 48 (91\%) hombres, edad $48 \pm 13$ años, la mayoría con miocardiopatía dilatada, 23 (43\%) e isquémica, 23 (43\%). Todos los pacientes, salvo 3 , se operaron por insuficiencia cardiaca: en 2 la indicación fue por tormentas arrítmicas y 1 por angina intratable. Alrededor de la mitad de los pacientes eran grupo $\mathrm{O}$ y un cuarto grupo A (Figura 1).

Cincuenta y tres por ciento (28 pacientes) estaban en urgencia (Estatus IA o IB), de los cuales $12(24 \%)$ estaban en asistencia ventricular mecánica (Figura 2). Todos los pacientes en asistencia estaban conectados a un Levitronix Centrimag salvo uno que estaba conectado a un Heartmate II. Fue pre-requisito para los pacientes en asistencia ventricular el rehabilitarse y ojalá levantarse de la cama previo al trasplante (Figura 3). El tiempo de espera promedio fue de $70 \pm 83$ días, con un
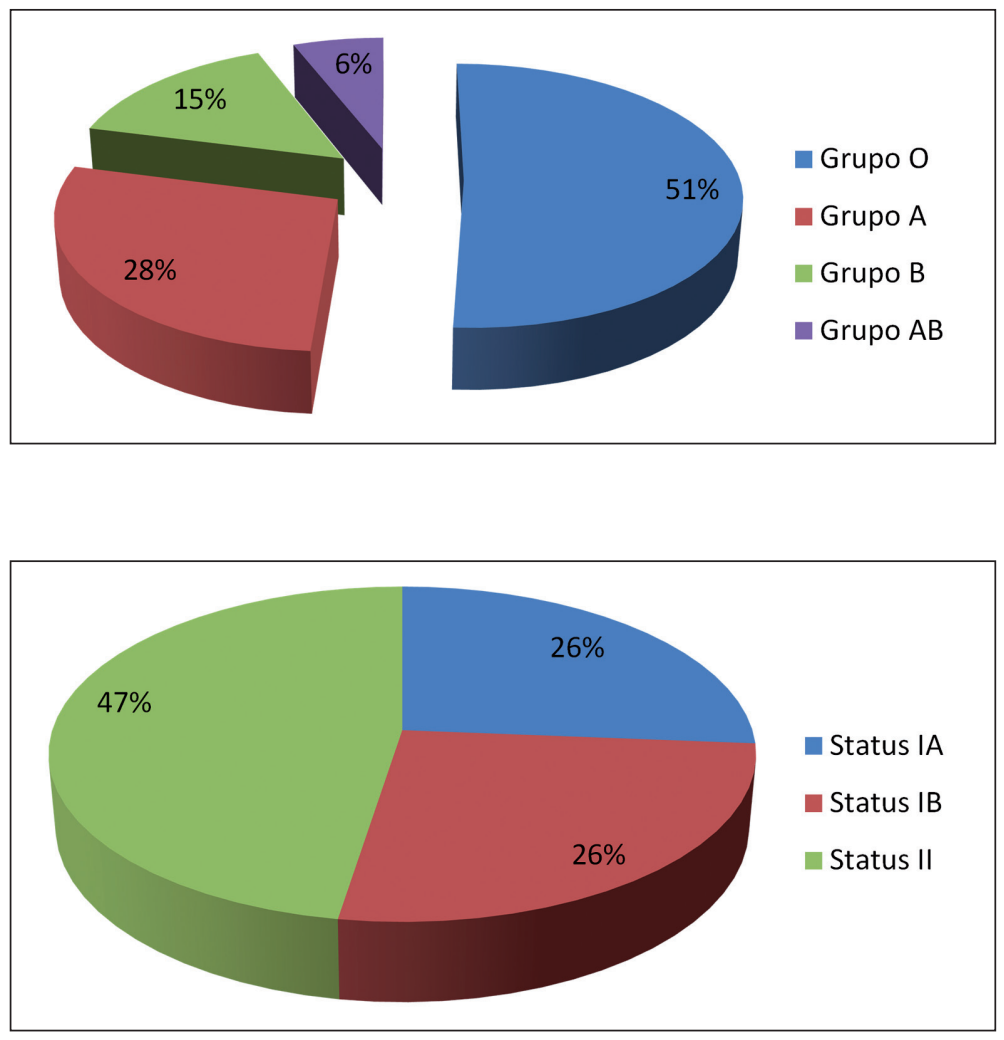

Figura 1. Distribución según grupo sanguíneo de receptores de trasplante.

Figura 2. Distribución de prioridad de trasplante. 


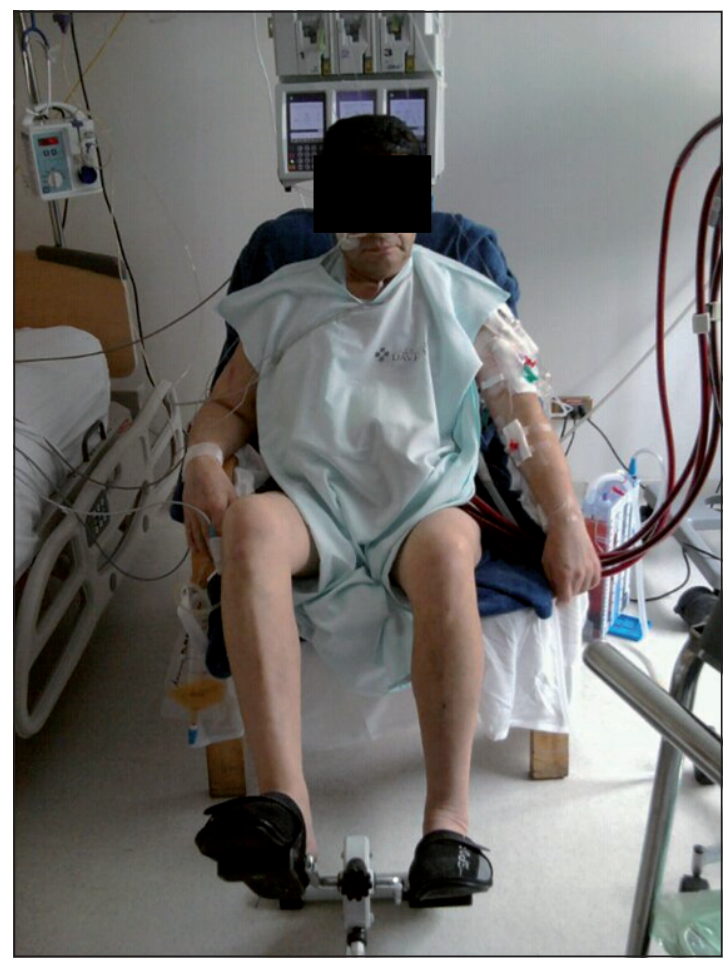

Figura 3. Paciente en asistencia ventricular rehabilitándose pre-trasplante.

mínimo de 0 y un máximo de 11 meses. En Estatus IA, la espera fue $29 \pm 25$ días, en Estatus IB $19 \pm$ 14 días y en Estatus II $122 \pm 96$ días.

En relación a los donantes, $80 \%$ (42 pacientes) estaba con noradrenalina con una dosis promedio de $0,2 \pm 0,1 \mathrm{mcg} / \mathrm{kg} / \mathrm{min}$ y $6 \%$ ( 3 pacientes) estaban con dopamina. En relación a la causa de muerte encefálica, 37 (70\%) fue por traumatismo encéfalocraneano y $14(26 \%)$ por un accidente vascular encefálico (AVE). Abuso de alcohol había en 15 (29\%), tabaco $21(40 \%)$ y cocaína 10 (19\%). Diez y ocho (34\%) eran de fuera de la Región Metropolitana.

La mortalidad operatoria fue de 3 pacientes (6\%). Todos ellos fallecieron por disfunción ventricular derecha que requirió "oxigenador de membrana extracorpórea” (por sus siglas en inglés: ECMO) en 2 y asistencia ventricular derecha en 1. Sin embargo, no lograron recuperarse. En un paciente se hizo evidente una hipoplasia severa de las arterias pulmonares que condicionó la disfunción ventricular y en los otros dos no se encontró un factor desencadenante claro. Otro paciente fue conectado a ECMO post-paro cardiaco en cuida- dos intensivos. Se recuperó y fue dado de alta con función biventricular normal. El tiempo promedio de estadía hospitalaria post-trasplante fue de $19 \pm$ 14 días y en cuidados intensivos $12 \pm 9$ días.

Morbilidad perioperatoria: hemorragia con re-exploración $2(4 \%)$, diálisis aguda $6(11 \%)$ y neumonía $4(8 \%)$. No hubo mediastinitis ni infección superficial de herida operatoria. Un paciente $(2 \%)$ requirió un marcapasos definitivo. En $3(6 \%)$ se produjo un AVE, uno falleció y otros 2 se recuperaron ad integrum. El paciente que falleció estuvo en ECMO por falla ventricular derecha post-trasplante y los otros dos estaban en asistencia ventricular pre-trasplante.

El seguimiento se completó en $100 \%$ de los pacientes, con un total de 1.091 meses de seguimiento. El tiempo promedio de seguimiento fue de $21 \pm 14$ meses, una variación de 1 a 54 meses y la mediana 21 meses. La sobrevida según KaplanMeier fue de $92 \pm 4 \%$ al año y $86 \pm 6 \%$ a los 3 años de seguimiento (Figura 4). Dos pacientes (4\%) fallecieron en el seguimiento, uno por neumonía por Pneumocystis jirovecii a los 9 meses y otro por abandono de los controles con muerte súbita a los 29 meses. Todos los sobrevivientes se encontraban en capacidad funcional I de NYHA en el momento del seguimiento y todos salvo uno tenían función biventricular normal.

Por protocolo se realizaron 484 biopsias endomiocárdicas. En ellas se encontró rechazo $2 \mathrm{R}$ que requirió terapia ad hoc en $11(2,3 \%)$. En todos, el tratamiento fue bolos de metilprednisolona y en dos pacientes se debió agregar plasmaféresis/ rituximab por rechazo humoral concomitante. Rechazo grado 0 hubo en 356 biopsias (74\%) y rechazo grado $1 \mathrm{R}$ en 117 (24\%). Todos los pacientes con biopsia grado $1 \mathrm{R}$ fueron asintomáticos por lo que sólo se optimizó la terapia inmunosupresora, pero no se utilizó terapia antirechazo específica. La sobrevida libre de rechazo con requerimiento de tratamiento específico fue de $90 \pm 4 \%$ a un año y $80 \pm 7 \%$ a 3 años.

Otras complicaciones en el seguimiento fueron: infección urinaria con requerimiento de antibióticos endovenosos en 4 (9\%), diarrea por micofenolato en $3(6 \%)$, stents a arterias coronarias en $2(4 \%)$, abscesos hepáticos en $1(2 \%)$, cáncer de piel en $1(2 \%)$, encéfalo-oftalmitis herpética, $1(2 \%)$, herpes zoster, $1(2 \%)$, enfermedad por citomegalovirus en $2(4 \%)$, y trombosis venosa profunda en $1(2 \%)$. 


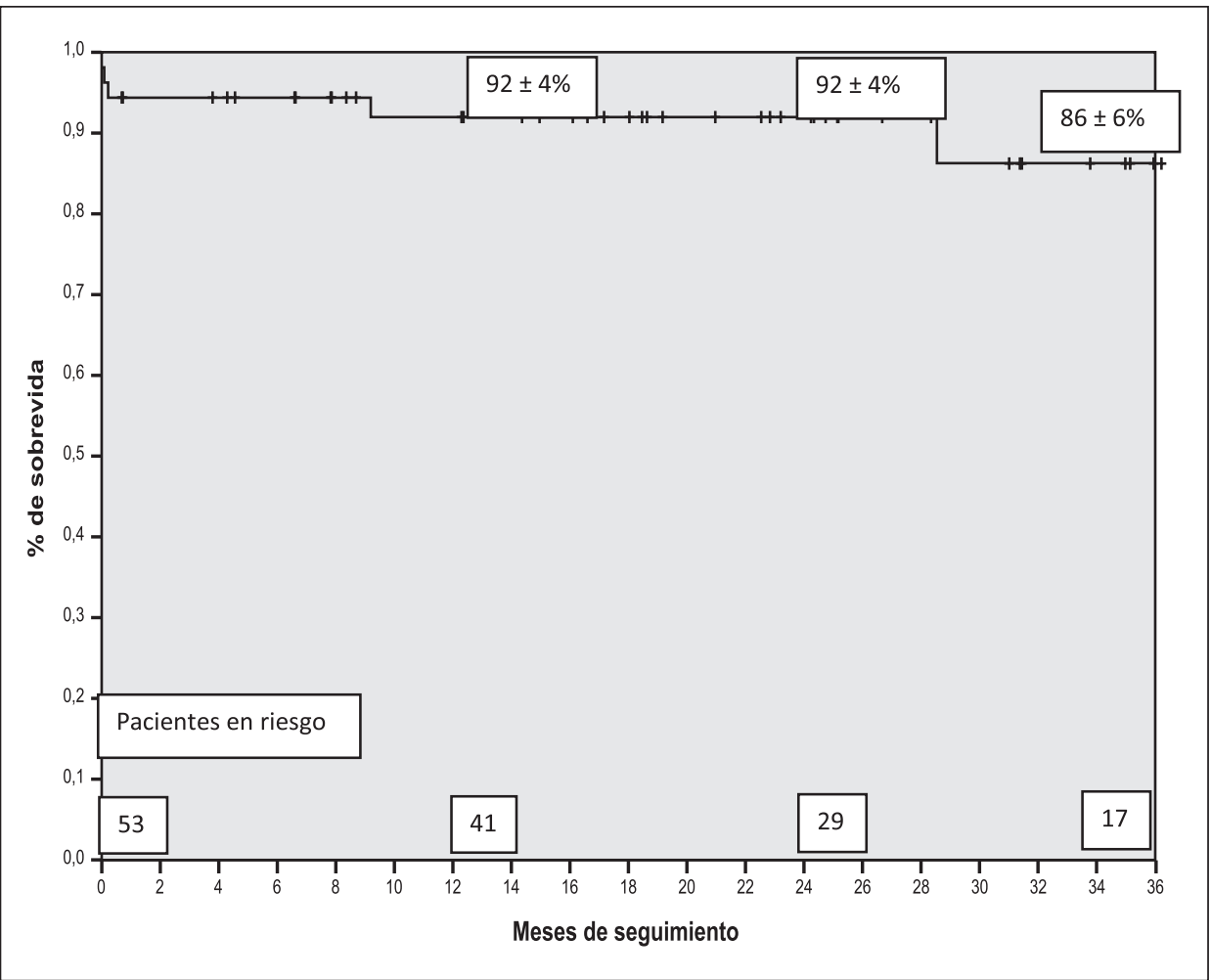

Figura 4. Sobrevida de 53 pacientes trasplantados de corazón.

\section{Discusión}

El trasplante cardiaco es la terapia de elección para la insuficiencia cardiaca avanzada que no responde a fármacos y/o a terapia de resincronización. Tal como ocurre en gran parte del mundo, el número de casos de trasplante cardiaco en Chile se ha mantenido estable en los últimos años. Entre los años 2004 y 2010 se realizaron un promedio de 19 trasplantes cardiacos por año a nivel nacional, con un máximo de 21, el año 2004 ${ }^{1}$. Esto origina que la tasa de trasplantes en Chile sea de 1,2 por millón de habitantes, muy lejos de la tasa de 7,7 por millón de habitantes de Estados Unidos de Norteamérica (USA) ${ }^{5}$. Posibles factores para explicar esta diferencia son problemas en el sistema de procuramiento, insuficiencia de una red de atención y derivación de pacientes con insuficiencia cardiaca, escasez de recursos para financiar los trasplantes, ausencia de dispositivos de asistencia ventricular y criterios demasiado exigentes para la elección de los donantes/receptores.

Sin embargo, en el año 2011 se produjo un aumento de 20 a 32 trasplantes (60\%), que puede tener que ver con cambios en la política gubernamental. El año 2011 entró en funciones operativas la Coordinadora Nacional de Trasplantes, dependiente de Ministerio de Salud y que reemplazó en sus funciones de procuramiento a una organización no gubernamental: la Corporación del Trasplante. Por primera vez el estado asumió la responsabilidad administrativa de la red de coordinadores de procuramiento a nivel nacional estableciendo metas sanitarias en relación de donantes y trasplantes de distinto tipo. Este es un primer factor que pudo haber incidido en el aumento a 32 en el número de trasplantes cardiacos en el 2011.

En segundo lugar, nuestro grupo cardioquirúrgico contribuyó con esta serie de 53 trasplantes cardiacos a $51 \%$ de la casuística realizada a nivel nacional durante igual período de tiempo (104 trasplantes totales). Una práctica distintiva de nuestro grupo fue no rechazar ningún órgano compatible que tuviéramos la posibilidad de evaluar in situ en el hospital del donante, particu- 
larmente aquellos que estaban en la Región Metropolitana y por ende, con un acceso más fácil. Esto queda de manifiesto con $86 \%$ de los donantes con drogas vasoactivas y con altos porcentajes de abuso de alcohol/drogas que en otras circunstancias se habrían desechado para trasplante.

Otra característica distintiva de nuestro grupo de trabajo fue la utilización de dispositivos de asistencia ventricular para lograr un puente a trasplante en $24 \%$ de la serie. Estos fueron pacientes en shock cardiogénico y falla multiorgánica que de otro modo no habrían logrado trasplantarse. La conducta clínica fue conectar al paciente a asistencia ventricular, esperar la recuperación de la falla multiorgánica y luego proceder al trasplante ${ }^{6}$. Si bien en el pasado hubo otra experiencia chilena con dispositivos de asistencia, esta no se mantuvo en el tiempo y durante el período de tiempo de esta serie sólo conocimos de casos esporádicos con esta técnica ${ }^{7}$.

Esta serie tiene un porcentaje más bajo de pacientes en asistencia ventricular que lo que muestra el registro de la ISHLT en la época más reciente ${ }^{2}$. En el año 2005, el porcentaje de trasplantados en asistencia ventricular era de $23 \%$, pero ya en el año 2010 alcanzó 36\%. Es de esperar, entonces, que en nuestro país se produzca un fenómeno parecido. Afortunadamente, en nuestra serie la mortalidad no se dio en pacientes conectados a asistencia, aunque sí hubo dos casos que tuvieron AVEs que se recuperaron ad integrum. Estos casos nos llevaron a una modificación en la conducta quirúrgica en cuanto a "clampear" precozmente la aorta antes de manipular las cánulas de asistencia y así prevenir embolias trombóticas. Después de esta modificación no tuvimos más accidentes neurológicos.

Es conocido en la prensa que existen esfuerzos por aumentar el número de donantes a nivel nacional, Chile tiene una tasa que bordea los 7 donantes por millón de habitantes, a diferencia de lo que sucede con el país líder en el planeta, España, que se acerca a los 36 por millón. No obstante, este problema no parece ser una limitante en el caso del trasplante cardiaco en nuestro país y en esta serie. En la serie a nivel global, el tiempo de espera promedio es de 70 días. En el caso de los pacientes urgentes el tiempo de espera varía entre los 19 y 29 días (IB y IA, respectivamente). Se infiere entonces que la disponibilidad de donantes no parece ser un problema mayor. El uso de dispositivos de asistencia ventricular de larga duración (Ej. Heartmate II o Heartware) no parece entonces justificado por el tiempo de espera en nuestro país en adultos para puente a trasplante. Esto en contraste con lo que sucede en Estados Unidos de Norteamérica con un largo período de espera que se acerca en promedio a los 12 meses $^{8}$. Podría justificarse el uso de estos dispositivos para acortar la estadía hospitalaria, ya que les permite a los pacientes manejarse ambulatoriamente y recuperarse más fácilmente.

En cuanto a la sobrevida de $86 \pm 6 \%$ a 3 años plazo, es alentadora si se la compara con la del registro de la ISHLT que muestra cerca de 78\% de los pacientes vivos durante el mismo seguimiento ${ }^{2}$. En el registro, la principal causa de muerte durante los 30 primeros días es la disfunción primaria del injerto, que es responsable de $64 \%$ de las muertes. En nuestra serie, los 3 pacientes que fallecieron tuvieron disfunción primaria del injerto. Más tardíamente aparecen en el registro las muertes por infección lo que coincide con nuestro paciente que falleció a los 9 meses por Pneumocystis jirovecii.

Durante el primer año post-trasplante cardiaco, 30-40\% de los pacientes tienen algún tipo de rechazo, que en la mayoría de los casos es asintomático, por lo que se justifica un protocolo se biopsias endomiocárdicas ${ }^{9,10}$. En nuestra serie, los resultados obtenidos son similares a los del registro internacional. Diecinueve por ciento de los pacientes tuvo rechazo moderado (11 biopsias $2 \mathrm{R}$ en 10 pacientes), que se compara con $16 \%$ del registro internacional. No parece entonces que tenga influencia negativa el uso de inmunosupresión genérica en trasplante cardiaco. De hecho, la sobrevida libre de rechazo es semejante a la del último período del registro internacional que es mejor que la de épocas pasadas.

Conclusión: Los resultados a mediano plazo son similares a los reportados por la ISHLT. Esta experiencia tiene una proporción más alta de pacientes urgentes y en asistencia ventricular que series nacionales previas. Las tasas de rechazo son bajas aun cuando se usó inmunosupresión genérica.

\section{Referencias}

1. www.trasplante.cl

2. Stehlik J, Edwards LB, Kucheryavaya AY, Benden C, Christie JD, Dipchand AI, et al. The Registry of the International Society for Heart and Lung Transplantation: 
Trasplante cardiaco - M. Villavicencio et al

29th official adult heart transplant report-2012. J Heart Lung Transplant 2012; 31 (10): 1052-64.

3. Aránguiz-Santander E, Merello L, Pedemonte O, Torres $\mathrm{H}$, Vera A, Alburquerque J. Heart transplantation in Chile: preliminary report from the Gustavo Fricke Hospital in Viña del Mar. Transplant Proc 2007; 39 (3): 619-21.

4. Morán S, Castro P, Zalaquett R, Becker P, Garayar B, José $\mathrm{M}$, et al. [Treatment of advanced heart failure by heart transplantation]. Rev Med Chile 2001; 129 (1): 9-17.

5. De Robertis F, Birks EJ, Rogers P, Dreyfus G, Pepper JR, Khaghani A. Clinical performance with the Levitronix Centrimag short-term ventricular assist device. J Heart Lung Transplant 2006; 25 (2): 181-6.

6. Shuhaiber JH, Jenkins D, Berman M, Parameshwar J, Dhital K, Tsui S, et al. The Papworth experience with the Levitronix Centri Mag ventricular assist device. J Heart Lung Transplant 2008; 27 (2): 158-64.

7. Villavicencio M, Larrea R, Larraín E, Turner F, Rivera J, Peralta JP, et al. [Four-week Levitronix Centrimag bridge-to-transplant for post myocardial infarction cardiogenic shock. A case report]. Rev Med Chile 2010; 138 (6): 752-7.

8. Aaronson KD, Slaughter MS, Miller LW, McGee EC, Cotts WG, Acker MA, et al. Bridge to Transplant ADVANCE Trial Investigators. Use of an intrapericardial, continuousflow, centrifugal pump in patients awaiting heart transplantation. Circulation 2012; 125 (25): 3191-200.

9. Strueber M, O’Driscoll G, Jansz P, Khaghani A, Levy WC, Wieselthaler GM; Heart Ware Investigators. Multicenter evaluation of an intrapericardial left ventricular assist system. J Am Coll Cardiol 2011; 57 (12): 1375-82.

10. John R, Kamdar F, Eckman P, Colvin-Adams M, Boyle A, Shumway S, et al. Lessons learned from experience with over 100 consecutive Heart Mate II left ventricular assist devices. Ann Thorac Surg 2011; 92 (5): 1593-9; discussion 1599-600.

11. Griffith BP, Kormos RL, Borovetz HS, Litwak K, Antaki JF, Poirier VL, et al. Heart Mate II left ventricular assist system: from concept to first clinical use. Ann Thorac Surg 2001; 71 (3 Suppl): S116-20; discussion S114-6.

12. www.unos.org

13. Castro P, Baraona F, Baeza C, Mc-Nab P, Berlin A, Zalaquett $\mathrm{R}$, et al. [Ventricular assist device as a bridge to transplant in patients with cardiogenic shock. Preliminary experience in Chile with ABIOMED BVS 5000]. Rev Med Chile 2006; 134 (8): 1019-23.

14. Kirklin JK, Naftel DC, Kormos RL, Stevenson LW, Pagani FD, Miller MA, et al. The Fourth INTERMACS Annual Report: 4,000 implants and counting. J Heart Lung Transplant 2012; 31 (2): 117-26.

15. Pham MX, Teuteberg JJ, Kfoury AG, Starling RC, Deng MC, Cappola TP, et al. Gene-expression profiling for rejection surveillance after cardiac transplantation. $\mathrm{N}$ Engl J Med 2010; 362 (20): 1890-900.

16. Stehlik J, Starling RC, Movsesian MA, Fang JC, Brown $\mathrm{RN}$, Hess ML, et al. Utility of long-term surveillance endomyocardial biopsy: a multi-institutional analysis. J Heart Lung Transplant 2006; 25 (12): 1402-9. 